\title{
REIFICAZIONE: DALLA LOTTA DI CLASSE ALLA LOTTA PER IL RICONOSCIMENTO
}

\author{
Irene Strazzeri \\ Università degli Studi di Foggia, Italia
}

http://dx.doi.org/10.5209/rev_NOMA.2013.v37.n1.42565

\begin{abstract}
La presente riflessione intende ridefinire la categoría della reificazione a partire dalla rivisitazione di questo concetto recentemente proposta da Axel Honneth. E' interessante sottolineare come Honneth riesca a collegare la dis-individuazione soggettiva con la mercificazione oggettiva, posta alla base delle tesi di Lukàcs e dunque la lotta per il riconoscimento con la lotta di classe. Infine si propone una ricomposizione delle due dimensioni della lotta nelle relazioni di cura.
\end{abstract}

Parole chiave.- lotta, riconoscimento, cura

\section{Reification: from class struggle to struggle for recognition}

Abstract.- This article redefines category of Reification starting from the actualization of this concept recently proposed by Axel Honneth. It's interesting to outline how Honneth is able to connect subjective dis-identification with the objective reification, located at the base of Lukàcs 's theses and therefore struggle for recognition with class struggle. Finally, the article proposes a shift of the two dimensions of struggle in care's relations.

Keywords.- struggle, recognition, care

\section{Introduzione}

Negli anni '20 e '30 del secolo scorso, la reificazione ha rappresentato il leitmotiv della critica sociale e culturale tedesca. In questo concetto si concentravano, come riflesse, le esperienze storiche della Repubblica di Weimar: la crisi economica da una lato e la forte disoccupazione dall'altro. Nel 1923 György Lukács, pubblicava l'opera Storia e coscienza di classe ${ }^{1}$, combinando i contributi di Marx, Weber e Simmel nell'ipotesi chiave che le forme della vita moderna fossero il risultato di processi di reificazione sociale. Dopo la II guerra mondiale la centralità della categoria venne meno, benché, con l'Olocausto, la diagnosi delle patologie sociali della modernità sia sopravvissuta nella Scuola di Francoforte e nella ripresa di Lukács da parte dei movimenti sociali, alla fine degli anni ' 60 . Oggi sembrerebbe che l'attualità della categoria della reificazione sia destinata a mutare ancora una volta. Alcuni indizi di questa ri-attualizzazione si possono incontrare in una serie di romanzi

\footnotetext{
${ }^{1}$ G. Lukács., Geschichte und Klassbewusstsein, tr. it. di Giovanni Piana; Storia e coscienza di classe, Sugarco ed., Roma 1978.
} 
che descrivono la penetrazione dei valori economici nella vita quotidiana come stato d'animo e come atmosfera sempre più diffusa (Raymond Carver, Harold Brokley, Michel Houellebecq con "L'enfant terribile", Elfriede Jelinek, Silke Scheuermann) e in una serie di ricerche sociologiche che identificano la reificazione attuale con quel comportamento umano, in cui determinati sentimenti e desideri vengono ostentati per ragioni opportunistiche, come se si trattasse di elementi autentici della propria personalità, una sorta di automanipolazione emotiva o management dei sentimenti. Si pensi al concetto di "objectification", attraverso cui Martha Nussbaum ${ }^{2}$ discute le forme estreme della strumentalizzazione di sé e degli altri come forme proprie di alienazione economica della vita contemporanea. Si parla, dunque, di reificazione in senso normativo, intendendo quel tipo di comportamento umano che viola determinati principi etici e morali in quanto si rapporta ai soggetti come merci, come cose e non come umani.

Si possono segnalare, infine, alcuni fenomeni empirici di riferimento: le maternità sostitutive, la "liquidità" delle relazioni amorose, ${ }^{3}$ lo sviluppo di una vera e propria industria del sesso. E ancora i risultati e le implicazioni delle ricerche fisio-biologiche sul cervello, intese come astrazioni su base neuronale dalle concrete esperienze sociali, come se l'essere umano fosse un automa privo di vita interiore. Un comportamento reificante è ritenuto tale, insomma, perché viola certi principi etici e morali, e non ontologici dell'agire umano, come invece riteneva Lukács. Ovviamente anche la reificazione di Lukács aveva un contenuto normativo, già il termine reificazione da il senso di una deviazione da un atteggiamento adeguato rispetto al mondo. Ma Lukács agisce per rappresentare la necessità storica della rivoluzione. Egli non vede, infatti, la deviazione etico-morale, ma solo quella connessa ad un certo tipo di prassi e razionalità. Si può dire che gli argomenti rivolti contro la reificazione capitalistica hanno solo indirettamente carattere normativo, poiché derivano da un'ontologia sociale che cerca di cogliere i fondamenti razionali della nostra esistenza. Lukács dunque consente di spiegare una determinata patologia della nostra prassi vitale. Ma non è così scontato giustificare le obiezioni contro una determinata forma di vita esclusivamente sulla base del punto di vista dell'ontologia sociale.

\section{La reificazione in Lukàcs}

Facendosi orientare dall'analisi classica di Lukács, si può notare come i suoi strumenti categoriali siano insufficienti ad un'adeguata concettualizzazione di dinamiche, spesso colte in modo corretto da un punto di vista fenomelogico. Lukács, si attiene alla concezione ontologizzante della reificazione, per cui reificazione non significherebbe altro che "rapporto tra persone che riceve il

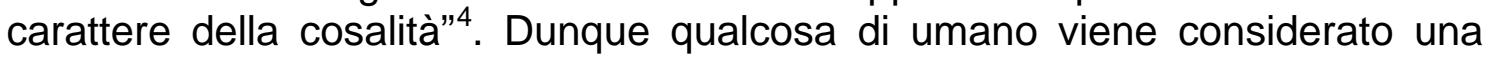
cosa. La reificazione sembrerebbe essere per Lukàcs un mero errore categoriale, epistemico, un atto moralmente riprovevole, costituito da una forma di prassi totalmente distorta.

\footnotetext{
${ }^{2}$ M. Nussabaum., "Objectification", in Sex and Social Justice, Oxford University Press 2000.

3 Z. Bauman., Modus Vivendi. Inferno e Utopia del mondo liquido, tr. it. di Savino D'Amico, Laterza, Bari 2007.

${ }^{4}$ G. Lukács, Storia e coscienza di classe, op. cit. p. 108
} 
Ma essa presenta "fenomenologicamente" una multidimensionalità e una stabilità che non possono essere spiegate come mero errore categoriale. La causa del persistere della reificazione, ad esempio, è in Lukàcs connessa all'espansione dei rapporti di mercato, diventati ormai la modalità prevalente dell'agire intersoggettivo. Non appena gli attori sociali cominciano a regolare le loro relazioni reciproche attraverso lo scambio di merci sono costretti a porsi in modo reificante rispetto al loro ambiente, e a percepire gli oggetti soltanto in riferimento alla loro utilità. Nello scambio di merci, in particolare, i soggetti sono reciprocamente indotti a percepire gli oggetti dati soltanto come cose da cui trarre profitto, considerarsi reciprocamente oggetto di una transizione vantaggiosa, considerare le loro risorse opportunità di profitto. Ma Lukàcs sussume tutti questi cambiamenti nel concetto di reificazione senza tenere conto delle molte possibili sfumature. Egli infatti designa "cosale" contemporneamente: la valutazione quantitativa degli oggetti, il trattamento strumentale di altre persone, la percezione del proprio talento, l'egoismo bello e buono, l'affermazione di un interesse puramente economico.

Ma come ha avuto luogo questa generalizzazione sociale? La tesi centrale di Lukács, è che "nel capitalismo la reificazione sia diventata la seconda natura dell'uomo" ${ }^{5}$. Tuttavia l'applicazione della coralità indifferenziata a tutti quei fenomeni non è sufficiente a giustificare l'idea di reificazione come seconda natura, riferendosi essa anche a sfere e dimensioni dell'agire sociale non economiche. Lo stesso Lukács, si è accorto di questo problema, tanto che, anziché guardare i cambiamenti negli oggetti percepiti, egli rivolge lo sguardo ai cambiamenti che il soggetto percepisce in se stesso. Sotto la coazione allo scambio, l'attore diventa uno spettatore contemplativo di se, incapace di influire su ciò che accade nella sua esistenza. II processo della reificazione si produce nella sfera d'azione, sempre più ampia, dello scambio di merci tra i soggetti, la stessa in cui sono costretti a comportarsi come spettatori distaccati, piuttosto che come partecipanti attivi alla vita sociale, perché il calcolo dei benefici che possono trarre dagli altri, gli richiede un atteggiamento razionale e immune da ogni condizionamento emotivo. Questo tipo di atteggiamento, nell'ipotesi di Lukács, diventa una "seconda natura". La contemplazione e il distacco diventano la chiave di lettura per spiegare ciò che avviene a livello di agire sociale quando si produce la reificazione. Il soggetto, anziché, essere impegnato nella sua prassi vitale, è situato nella prospettiva di un osservatore neutrale. Questa è, secondo Axel Honneth, la base di spiegazione più adeguata della reificazione come oblio di riconoscimento ${ }^{6}$.

\section{La reificazione come oblio di riconoscimento in Honneth}

Come abbiamo visto, la reificazione non è e non può essere un puro e semplice errore categoriale o epistemico, ma una sindrome sociale di coscienza distorta a più livelli, poiché investe troppo profondamente le nostre abitudini per essere modificata con una correzione cognitiva. La reificazione è un atteggiamento che distorce la nostra prospettiva, tanto diffusa da poter essere considerata una seconda natura.

\footnotetext{
5 Ivi, p. 111.

6 A. Honneth., Verdinglichung, Suhrkamp Verlag, Frankfurt am Main 2005; tr. it. di Carlo Sandrelli, Reificazione, Meltemi, Roma 2005.
} 
Essa non è neppure una condotta moralmente sbagliata o una violazione di principi morali, perché in tale ottica non si potrebbe prendere in considerazione l'elemento essenziale dell'intenzionalità soggettiva, essenziale per poter mettere in gioco una terminologia morale. Se non è un errore categoriale, se non è una violazione morale, non rimane che concepirla, propone Honneth, come una forma di prassi strutturalmente falsa ${ }^{7}$. Ma questa definizione non è affatto priva di implicazioni normative, poiché induce ad un confronto con il compito molto più difficile di dimostrare l'esistenza di una prassi "vera" o "autentica", oltre e contro le prassi sociali distorte e atrofizzate. I suoi presupposti normativi non sono dati da una somma di principi moralmente legittimanti, ma dall'ipotesi/concetto di una giusta prassi umana ${ }^{8}$. Tale ipotesi ricaverebbe la sua giustificazione dagli assunti della filosofia morale o dall'etica sociale. Ma Honneth non intende privare la critica della reificazione di una qualche chance di giustificazione in termini di teoria sociale. Innanzitutto la prassi autentica che ha in mente possiede quelle caratteristiche di partecipazione e coinvolgimento, che non sono andate completamente distrutte nell'espansione dello scambio nelle relazioni sociali umane. II modello sarebbe costituto "da un atteggiamento intersoggettivo che manifesta caratteri opposti rispetto alla prassi reificante" 9 . Seguendo questa traccia, Honneth si domanda se abbia senso riattualizzare il concetto di "reificazione" nel confronto con una prassi intersoggettiva originaria più autentica, nella quale gli uomini stabiliscano una relazione simpatetica tra di loro e con il loro ambiente. Di qui, a mio avviso, l'autore consente sviluppi illuminanti, dovuti alla rilettura in chiave di riconoscimento di alcune categorie di Dewey "l'interazione", e di Heidegger "la cura".

II mio contributo tenterà di dimostrare come la cura possa rappresentare una risposta a quell'oblio di riconoscimento, che caratterizza i contemporanei processi di reificazione. Per sostenere questa tesi, dovremo però seguire Honneth, nel suo tentativo di dimostrare la priorità genetica e concettuale del riconoscimento sulla conoscenza.

Innanzitutto, in luogo delle variabili individuate da Lukács: l'espansione del mercato capitalista e la crescente mercificazione dei rapporti sociali, Honneth ci invita a considerare una diversa"eziologia sociale" della reificazione, tesa a dimostrare, appunto, che essa è soprattutto oblio di riconoscimento. Secondo Honneth l'aspirazione degli individui al riconoscimento intersoggettivo della propria identità è insita, fin dall'inizio, nella vita sociale come tensione ineliminabile. Com'è noto, all'idea di "lotta sociale", come elemento motore nell'autodeterminazione delle individualità, cara a buona parte della tradizione politica moderna (da Hobbes a Machiavelli), già Hegel conferì una caratterizzazione di enorme rilievo. La dinamica del riconoscimento, liberata dalle connotazioni trascendentali dell'idealismo fichtiano, consentiva una lettura dell'eticità dell'uomo orientata secondo successivi stadi di conciliazione e di conflitto. $^{10} \mathrm{E}$ ' stato dunque Hegel il primo ad intravedere la possibilità di

\footnotetext{
${ }^{7}$ A. Honneth., Reificazione, op. cit. p. 22.

${ }^{8}$ Ibid.

${ }^{9}$ A. Honneth., Reificazione, op. cit. p. 23.

${ }^{10}$ G.W.F. Hegel., Jenaer kritische Schriften, a cura di H. Buchner-O. Poeggeler, Bd. 4 der Gesammelten Werke, Meiner, Hamburg 1968., Cfr. Primi scritti critici, a cura di Remo Bodei, Mursia, Milano 1971.
} 
leggere, attraverso un unico registro, le esigenze di riconoscimento in rapporto alla conflittualità sociale. Le differenti modalità di riconoscimento che, secondo Honneth, Hegel sembra aver intuito sono quelle dell'amore, del diritto e della solidarietà, elementi normativi violati nelle esperienze di spregio e umiliazione, o meglio di misconoscimento, rispettivamente individuate nella violazione dell'integrità fisica, nel razzismo e nella negazione dei diritti fondamentali. Amore, diritto e solidarietà sono dunque modalità intersoggettive di riconoscimento, insite nei processi sociali di autorealizzazione personale.

Un altro aspetto sul quale le analisi di Honneth si soffermano, a conferma e completamento del nesso costitutivo integrità della persona /riconoscimento, è la possibilità di una fenomenologia empiricamente controllabile delle forme di riconoscimento $^{11}$, che si colloca nell'interazionismo simbolico di G. H. Mead. II riferimento a Mead consente ad Honneth di tradurre la teoria intersoggettivistica in un linguaggio teorico post-metafisico. Quello che emerge è che l'autore di Mente, sé e società ${ }^{12}$ condivide con Hegel non soltanto l'idea di una costruzione sociale della soggettività, ma che manifesti affinità ancora più profonde con Hegel, proprio riguardo alla concezione del riconoscimento. Com'è noto una delle intuizioni centrali del pensiero di Mead è la distinzione tra "io" ed "me". L'individuo, inoltre, nel suo continuo processo di formazione, si oggettiva in diversi "Se" distinti dall"'lo". Mead inoltre illustra le dinamiche attraverso le quali accediamo alla formazione di questi "Se" distinti dall' "io"?. I meccanismi di formazione del "Se" sono riconducibili fondamentalmente a processi di interazione sociale: solo a partire dalla distinzione tra Play e Game, diviene infatti possibile comprendere in quali forme. In ambedue queste modalità del gioco, il bambino produce il proprio "Me" ma, a differenza del Play ("il gioco puro e semplice"), dove l'interlocutore è una semplice proiezione dell' "lo", nel Game ("il gioco organizzato"), la proiezione deve interiorizzare l'atteggiamento di tutti gli altri partecipanti, deve presumere cioè la prospettiva di un "altro generalizzato". Insomma, nell'agire sociale, solo riconoscendo un altro generalizzato, possiamo concepire l'interazione sociale in modo normativo. Se per autorealizzazione intendiamo, con Mead, un momento di autoaccertamento etico della società, la soluzione da lui indicata, è quella secondo cui si può trovare un momento di conferma del proprio operato nell'assunzione piena del proprio ruolo sociale Alla luce di queste analisi, Honneth proporrà una sorta di modellistica del riconoscimento, in grado di distinguere l'amore, dal diritto dalla solidarietà. A partire dalla concezione hegeliana dell'amore come "esser se stessi in un estraneo" e combinando quest'ultima con le tesi sull'intersoggettività primaria di Winnicott, Honneth dimostra come l'amore possa rappresentare un primo stadio di riconoscimento. L'amore, soprattutto quello esperito nelle relazioni intersoggettive di tipo primario, rappresenta il "nucleo originario di ogni eticità" ed è solo a partire da esso, che le sfere del diritto e della solidarietà, vengono ad istituzionalizzarsi. Nell'amore, infatti, sarebbero incluse quelle forme di relazione tra io e me, in cui si rispecchiano

\footnotetext{
${ }^{11}$ A. Honneth., Kampf für Anerkennung. Grammatik sozialer Konflikte, Suhrkamp Verlag, Frankfurt a.M. 1992; tr. it di Carlo Sandrelli, Lotta per il riconoscimento. Proposte per un'etica del conflitto, il Saggiatore, Milano 2003.

${ }^{12}$ Mind, Self and Society. From the Standpoint of a Social Behaviorist, University of Chicago Press, Chicago 1934; tr. it. di Roberto Testucci, Mente, Sè e Società, Giunti-Barbera, Firenze 1966.
} 
vicendevolmente gli attori sociali, perché possiedono una comune conoscenza del contesto e delle norme in cui sono immersi. Dal reciproco riconoscimento dei soggetti deriva, in ultimo, la stima sociale, una forma di riconoscimento valoriale ed affettivo dell'altro, che trova nell'idea di solidarietà il proprio compimento formale. Quali sono, invece, gli effetti prodotti dall'assenza di riconoscimento? Abbiamo visto, poco prima, come la reificazione, in quanto incapacità di tener conto del momento controbilanciante del riconoscimento intersoggettivo nella formazione dell'identità personale, possa indurre gli individui a "disimparare" la dimensione del riconoscimento, che è invece costitutiva di tutte le relazioni sociali nelle quali la persona è implicata.

Un esempio mondano ma chiarificatore di questo tipo di reificazione potrebbe essere rappresentato da una partita di tennis, i cui giocatori desiderino così intensamente la vittoria, da perdere il senso di "tutti gli altri possibili e più originali motivi e scopi"della partita"13. il fatto che il proprio avversario possa essere un amico o il fatto che una ricompensa all'amicizia, meno egoistica, potrebbe semplicemente consistere nell'originalità del proprio stile di gioco. Tale circostanza rappresenterebbe un caso paradigmatico di reificazione, poiché il nostro giocatore di tennis avrebbe perso di vista la previa relazione di riconoscimento con l'amico/avversario, che è anche la condizione di possibilità della situazione presente -lo scopo finale della vittoria domina così tanto la sua attenzione al mondo che tutte le caratteristiche di esso diventano irrilevanti per lo scopo finale e dunque vengono "obliate". Altri esempi paradigmatici di reificazione intersoggettiva, il razzismo, il sessismo, il traffico di esseri umani, lo sfruttamento lavorativo, appartengono invece a quella tipologia, in cui, secondo Honneth, "I'attenzione alla priorità del riconoscimento si perde a causa del fatto che ci lasciamo influenzare da pregiudizi e schemi concettuali, cognitivamente inconciliabili con quella priorità"14.

A partire dalla definizione di reificazione, come oblio del riconoscimento, citerò adesso i successivi sviluppi sociali di tipo reificante individutati da Honneth piuttosto che da Lukàcs, per chiarire come essi potrebbe trovare risposta in un'accezione della cura, quale prassi sociale caratterizzata dal coinvolgimento esistenziale ed emotivo fondato sul riconoscimento. I casi potrebbero essere rappresentati dal crescente svuotamento della sostanza legale dei contratti di lavoro; da tutte quelle pratiche per le quali, i talenti potenziali dei bambini hanno cominciato a essere considerati soltanto come fattori di misurazione genetica e di manipolazione, dall'istituzionalizzazione di pratiche di presentazione del sé orientate in senso "funzionalistico", dal cosiddetto lavoro emotivo, ossia la condizione di percepire certi sentimenti come parte integrante della propria attività (durante i colloqui di lavoro, le professioni di hostess, i servizi di dating). In tutte queste situazioni, viene obliata una precedente situazione di riconoscimento, viene cioè negata la priorità genetica della dimensione del riconoscimento sulla conoscenza.

\footnotetext{
${ }^{13}$ Honneth, Reificazione, op. cit. p. 71.

${ }^{14}$ Ivi, p. 72
} 


\section{La priorità genetica del riconoscimento}

La tesi della priorità genetica del riconoscimento potrebbe trovare conforto nell'evidenza per la psicoanalisi, e in genere per la psicologia evolutiva, del fatto che la capacità del bambino di relazionarsi al mondo esterno di oggetti stabili e costanti è prioritariamente condizionata dalla sua capacità di assumere il punto di vista di una seconda persona. Tale capacità dipende, a sua volta, dalla qualità emotivamente non disturbata della relazione del bambino con coloro che all'inizio si prendono cura di lui. Alcuni esempi potrebbero confortare questa tesi:

- il caso dei bambini autistici ${ }^{15}$ : come risulta dall'analisi del comportamento dei bambini autistici, quando, per incapacità di colui che si prende cura di loro o per altre ragioni, il bambino non riesce a sviluppare un rapporto affettivo con la figura dell'adulto e non riesce a "essere spinto, motivato e trascinato" dalla presenza di tale figura e, quindi, a identificarsi con una seconda persona, assumendo il suo modo di vedere il mondo, allora il bambino resta "emozionalmente cieco" e anche le sue capacità cognitive non riescono a svilupparsi. La visione impersonale del mondo di oggetti indipendenti deriva strettamente dalla dimensione "relazionale" e "personale" di una "apertura spontanea di dedizione o di amore". Pertanto, la "ricettività emotiva" ha "precedenza semplicemente temporale" rispetto alla capacità di conoscere "oggetti dati intersoggettivamente".

- la "nascita psicologica" dell'infante ${ }^{16}$ : Margaret Mahler ha recentemente proposto il concetto innovativo di nascita psicologica, sulla base della constatazione che il bambino ha bisogno di vivere un'effettiva seconda nascita dal punto di vista psicologico, la quale non è in alcun modo la conseguenza automatica della prima nascita biologica. Tale seconda nascita puó essere esperita dal bambino solo quando egli riesce a negoziare con successo la sua uscita dall'orbita simbiotica della fusione con una figura parentale.

- la psicologia del sé di Heinz Kohut ${ }^{17}$ offre un'ulteriore evidenza del fatto che, quando le esigenze primarie di imitazione e di identificazione non sono soddisfatte da figure parentali, il sé umano non riesce a svilupparsi, e i confini tra mondo esterno e rappresentazioni interne risultano sistematicamente sbiaditi. Kohut usa esplicitamente il linguaggio del riconoscimento: il bambino riesce a conquistare un'autonomia psicologica e a stabilire una giusta relazione con il mondo esterno soltanto se ha goduto di un riconoscimento incondizionato quale soggetto autonomo di azione da parte di una figura parentale primaria. $\mathrm{Se}$, al contrario, il bambino è stato soltanto oggetto delle fantasie narcisistiche e manipolative di un genitore incapace di riconoscimento, egli sarà allora condannato a oscillare tra la ricerca di un rispecchiamento incondizionato e l'identificazione ideale con figure onnipotenti.

\footnotetext{
15 M. Dornes., „Die Emozionale Ursprünge des Denkens“, in WestEnd. Neue Zeitschrift für Sozialforschung, a.2, n.1, pp. 3-48.

${ }^{16}$ M. Mahler et al., The Psychological Birth of the Human Infant: Symbiosis and the Vicissitudes of Individuation, New York, Basic Books 1975; La nascita psicologica del bambino, tr. it. di A.Guglielmi, Boringhieri, Torino 1978.

${ }^{17} \mathrm{H}$. . Kohut, The Restoration of the Self, New York, International University Press 1977; tr. it., La guarigione del sé, Bollati Boringhieri, Torino 1980 pp. 185-86.
} 


\section{La priorità concettuale del riconoscimento}

Da un punto di vista concettuale la priorità del riconoscimento si riferisce alla fatto che la comprensione del significato delle espressioni umane, siano esse verbali o extraverbali, richiede da parte nostra un atteggiamento di riconoscimento, soprattutto se la comprensione tra gli individui è legata al presupposto non-epistemico, ma genetico, del riconoscimento dell'altro. Si potrebbe semplicemente affermare, che non riusciremmo a capire interamente ció che gli altri ci dicono, o ciò che gli altri sentono ed esprimono, se non volessimo o non potessimo adottare un atteggiamento di riconoscimento. Questa convinzione è anche alla base degli studi di Stanley Cavell, il quale analizza la priorità del riconoscimento attraverso la critica dei tentativi tradizionali di confutare le tesi degli scettici circa la possibilità di accedere alle menti altrui. Ció che condanna al fallimento la tradizionale difesa antiscettica della possibilità di accedere agli stati emozionali altrui è il fatto che tale difesa è principalmente affrontata in termini cognitivi: rispetto alla conoscenza degli stati mentali altrui non possiamo infatti mai raggiungere lo stesso grado di certezza di quella che abbiamo nei confronti dei nostri propri stati mentali. La soluzione proposta da Cavell reputa che il modo migliore di stabilire l'esistenza delle menti altrui è quello di concepire la nostra relazione con una persona negli stessi termini nei quali concepiamo la relazione di quella persona con i suoi propri stati mentali: "così come in questo caso non parliamo di conoscenza, ma di implicazione o coinvolgimento, non dovremmo pensare chi agisce comunicativamente come un soggetto epistemico, ma come un soggetto esistenzialmente coinvolto, che non si limita a prendere atto in modo neutrale degli stati emotivi di altre persone, ma viene toccato da essi nelle sue stesse possibilita' autocomprensione" ${ }^{18}$.

Per dirla in termini più rozzi, il modo in cui possiamo raggiungere la certezza riguardo all'esistenza delle menti altrui è di essere consapevoli che noi amiamo alcune di queste menti mentre ne odiamo altre. Tornando al problema se sia mai possibile accedere agli stati emozionali altrui, Cavell sottolinea che colui che parla "attira l'attenzione di colui che ascolta" verso i suoi propri stati mentali in maniera diretta, "senza ricorrere alla conoscenza", e colui che ascolta partecipa con successo alla conversazione rispondendo con "simpatia" o consonanza piuttosto che con attestati di nuova conoscenza. Nello stesso modo, quando reagiamo a una espressione di dolore da parte di colui che parla, dicendo "so che tu soffri", non intendiamo comunicare il nostro "sapere" in senso cognitivo - il nostro sapere che cos'è il dolore - ma esprimere simpatia al nostro interlocutore: vogliamo solo comunicargli che sappiamo cosa vuol dire soffrire .

Essere un soggetto competente vuol dire quindi essere inserito in una rete di relazioni con gli altri, all'interno delle quali noi riceviamo l'espressione dei loro stati d'animo e le loro richieste non solo per testimoniare loro la comprensione cognitiva di quanto esprimono, ma anche per "reagire in maniera adeguata" ad esse. Cosa significa una "maniera adeguata" ? Adeguata è quella reazione che mostra che siamo consapevoli delle richieste che l'altro ci rivolge, che lo riconosciamo come una mente uguale alla nostra. Se non siamo capaci di

${ }^{18}$ S. Cavell., "Knowing and Acknowledging" in Must We Mean What We Say?,Cambridge University Press 1976, p. 238. 
assumere questo tipo di riconoscimento, vuol dire che siamo anche incapaci di far parte di quella rete di relazioni sociali. Da ció risulta un'importante conseguenza per la nostra comprensione della società. II tessuto delle interazioni sociali non è lo scambio di affermazioni cognitive, ma lo scambio di atti di riconoscimento reciproco. Come sostiene Honneth, ció che Cavell ci offre è la possibilità di andare al di là del semplice argomento della priorità del riconoscimento rispetto alla conoscenza.

Honneth tuttavia attira la nostra attenzione sul fatto che, per Cavell, la priorità del riconoscimento in quanto coinvolgimento esistenziale è rilevante soltanto rispetto al mondo sociale e non si applica al mondo naturale. Tutto ció del resto era ben noto anche alla filosofia analitica del linguaggio, si pensi a John Searle, il quale non si stanca di ripetere che ció che ci fa attribuire un significato a certi scritti su un foglio di carta e a certi suoni umani che sollecitano le mie orecchie è il presupposto che essi possano comunicarmi intenzionalmente gli stati intenzionali di un essere umano uguale a me. ${ }^{19}$

Le conclusioni di Honneth sono allora così riassumibili: la reificazione non è un errore categoriale (dal momento che si riferisce a un habitus o a un comportamento sociale) e non è un torto morale (dal momento che non puó essere ricondotta a un'istanza di responsabilità o di colpa). La reificazione è, al tempo stesso, un processo e un risultato: essa indica sia il prodursi di una perdita, cioè la sostituzione di una dimensione originaria e corretta con una secondaria e falsa - sia l'esito di un processo, vale a dire una percezione e un comportamento reificati.

\section{La cura come risposta alla reificazione}

Heidegger aveva indicato le cause della reificazione "nell'effetto deformante prodotto dalle immagini ontologiche del mondo", che indurrebbe gli individui alla "Seinsvergessenheit", ossia verso quella forma patologica di reificazione che è da lui definita "l'oblio dell'essere"20. Lukács era stato invece portato dal suo schema teorico a riferire la reificazione esclusivamente a cause sociali. In diversi punti della sua opera, come abbiamo visto, troviamo esposta la tesi secondo cui certe condizioni sociali - l'economia capitalista, lo sfruttamento del lavoro, la diffusione della logica del mercato - comportano una sorta di neutralizzazione della dimensione in sé prioritaria del riconoscimento e del coinvolgimento. Honneth non trova plausibile tale spiegazione, non ultimo per il fatto che quanto più si fa riferimento a un processo weberiano di razionalizzazione, tanto più si tende a considerare la reificazione come un fenomeno che investe tutti i settori della vita sociale, minando le condizioni stesse della sua riproduzione.

In contrasto con Lukács e attraverso Heidegger, Honneth non intende sviluppare un concetto di reificazione che conduca al risultato contro-intuitivo che ogni istanza di neutralizzazione e di oggettivazione - comprese quelle funzionali al mantenimento della vita e al suo sviluppo - sia automaticamente un fenomeno di reificazione che mina il tessuto sociale. Egli vorrebbe piuttosto

\footnotetext{
${ }^{20}$ M. Heidegger., Sein und Zeit (1927); Essere e tempo, a cura di Franco Volpi, tr. it. di Pietro Chiodi, Longanesi, Milano 2005.
} 
diminuire l'opposizione tra forme di conoscenza fondate sul riconoscimento e forme di conoscenza oggettivanti e, a questo fine, egli distingue tra due modi della loro relazione: nel primo caso la conoscenza, ovvero l'osservazione distaccata, avviene nella piena consapevolezza della sua dipendenza dal precedente riconoscimento, nel secondo caso, invece, respinge questa dipendenza e "si illude di essere autonoma da qualsiasi presupposto nonepistemico" ${ }^{21}$. A questo punto, la rielaborazione di Honneth e la sua riappropriazione del concetto di reificazione è conclusa:" la reificazione é meglio intesa come "oblio del riconoscimento", ovvero come perdita della consapevolezza di quanto il sapere e la conoscenza dipendano, per la loro esistenza, da una "precedente disposizione alla partecipazione coinvolta, alla cura e al riconoscimento" ${ }^{22}$. Le forme possibili di oblio di questa disposizione, infine, si manifesterebbero principalmente in tre modi:

1) rispetto ad altre persone, dimenticando il loro precedente riconoscimento

2) rispetto al mondo esterno, perdendo di vista la molteplicità dei possibili significati attribuiti ad esso dalle persone precedentemente conosciute

3) la "reificazione del se", l'inautenticità, ovvero la relazione con se stessi caratterizzata dall'oblio del riconoscimento del ruolo che gli altri hanno avuto nella formazione del se.

Proprio a partire dalla terza forma, mi sembra si possa impostare il discorso sulla cura, come contenitore e contenuto del riconoscimento. Bisognerebbe secondo chiedersi come sia possibile l'autoreificazione. Com'è possibile, ossia, che io abbia una relazione non-riconoscitiva con me stesso? Dalla psicologia evolutiva, come si è visto, è stata sottolineata la possibilità che una persona possa riferirsi alle sue proprie motivazioni senza realmente valutare il significato che esse assumono all'interno del sé, inteso come un tutto, cioè in modo inautentico, in quanto non si tiene conto del significato che le motivazioni assumono per l'insieme delle immagini e del progetto costitutivi del se. Non resta allora che ripercorrere gli sviluppi, sociali e scientifici, che hanno accompagnato la diffusione di pratiche di autoreificazione.

Da un lato, la persona puó assumere un atteggiamento puramente cognitivostrumentale verso il suo stesso sé , sulla base di ciò che David Filkenstein chiama "detectivist relation" ${ }^{23}$, che sta ad indicare una relazione primaria, soltanto cognitiva nei confronti del mondo esterno. Una concezione "detectivista" di se stesso, è una concezione nella quale l'irrinunciabile e costitutiva dimensione della "priorità del riconoscimento" viene intesa come una descrizione fattuale, vera della sua propria costituzione. Tra gli autori che hanno denunciato tale posizione, si potrebbe citare Searle, ma anche molte altre fonti importanti - da Pascal a Valéry, a Girard e Luhmann.

\footnotetext{
${ }^{21}$ A. Honneth., Reificazione, op. cit. p. 20.

22 Ibid.

${ }^{23}$ D. Finkelstein., Expression and the Inner, Cambridge, Harvard University Press 2003.
} 
All'altro estremo della relazione reificata con il proprio mondo interiore, sta la posizione che Honneth chiama "costruttivismo", ovvero l'idea che noi creiamo noi stessi, il nostro mondo interiore, soltanto attraverso la descrizione che facciamo di noi: nel momento in cui formuliamo determinate intenzioni di fronte ai nostri partner nell'interazione, in certo modo decidiamo di farle esistere in noi. Si tratta di una concezione puramente "costruttivista" del sé, nella quale la "priorità del riconoscimento" viene intesa come il risultato di un atto di scelta convalidato dal semplice fatto di selezionare e preferire tale descrizione come vera. Questo modello ipervolontarista della relazione con il sé della persona è, evidentemente, ugualmente dimentico del momento del riconoscimento.

Potremmo, allora, tentare di indicare una via di mezzo, nel carattere "espressivo" della relazione di cura, secondo la quale noi non percepiamo i nostri stati mentali semplicemente come oggetti, né li costituiamo grazie alle nostre espressioni linguistiche, ma li articoliamo in funzione di ciò che ci è interiormente già familiare. Un soggetto che si rapporta a se stesso in questo modo originario, deve considerare i propri sentimenti e i propri desideri come qualcosa che merita di essere articolato, come degni, meritevoli di cura. Tuttavia, sebbene anche per la cura si possa parlare di necessità di un riconoscimento antecedente, essa richiede una diversa comprensione della "priorità del riconoscimento", poiché Honneth tende a rappresentare tale comprensione come basata a sua volta, riflessivamente, sul riconoscimento. La sua giustificazione della "priorità del riconoscimento" dovrebbe, invece, necessariamente aver luogo, se non vogliamo cadere nella contraddizione performativa di un'istanza cognitiva, che verrebbe "scoprendo" la priorità del riconoscimento con atto di riconoscimento, nel contesto della "cura", data la dimensione "affermativa" e il "contatto espressivo" che in questa relazione il soggetto matura con se stesso e con la sua storia. Potremmo dire che ció che la "cura" e l' "affermazione" richiedono, al fine di ricostruire le caratteristiche di una prassi sociale non reificata e per rispondere alle esigenze della nostra costituzione intersoggettiva, è che il soggetto umano in questione formuli un giudizio riflettente riguardo a quale sia quella prassi che dal suo punto di vista meglio si adatta alla relazione di autoriconoscimento. Da tale punto di vista riflessivo, la priorità del riconoscimento rispetto alla conoscenza non puó essere intesa né come una scoperta né come un'illusione onnipotente di autocostruzione del sé, ma precisamente come un atto di cura e di affermazione. A questo punto, mi domando se non ci troviamo di fronte a ciò che Alessandro Ferrara definisce il ruolo imprescindibile del giudizio al servizio dell'ottimizzazione dell'identità ${ }^{24}$. Mi domando se la nostra relazione di cura e affermazione di noi non sia meglio valorizzata adottando il criterio della "priorità del riconoscimento rispetto alla conoscenza", essendo tale criterio, in ultima analisi, un atto di giudizio riflettente riguardo a ció che la relazione di cura richiede. Se le cose stanno cosí, avremmo scoperto che la dimensione del giudizio - riflessivo riguardo a ció che è più conveniente per la fioritura dell'umano - svolge un ruolo anche nei confronti della tesi della priorità del riconoscimento rispetto alla conoscenza. In questo senso, il soggetto reputa se stesso capace di entrare in un contatto espressivo non solo con i propri stati mentali, ma anche con i sentimenti dell'altro. Pertanto dovrà considerare i suoi

\footnotetext{
${ }^{24}$ A. Ferrara., La forza dell'esempio. II paradigma del giudizio, Feltrinelli, Milano 2008, p. 55.
} 
desideri e sentimenti e, in genere, le sue esperienze psichiche come degne di articolazione, sviluppando un atteggiamento positivo di cura e amore di sé.

La cura risulta in tal modo intimamente connessa ad un atteggiamento di sostegno, di conferma, in definitiva di riconoscimento della soggettività. Questo nesso categoriale tra un'autentica prassi umana, la cura, e un atteggiamento affermativo, il riconoscimento, è la condizione di possibilità di un rapporto autentico con se stessi. Si potrebbe così dimostrare non solo la priorità genetica e categoriale del riconoscimento rispetto alla conoscenza, come vorrebbe Honneth, ma anche l'intima connessione tra la dimensione sociale della cura, il riconoscimento intersoggettivo e la capacità riflessiva di giudizio degli attori, se posti in condizioni emancipate dalla minaccie di reificazione . 